\title{
Ketamine for chronic depression: two cautionary tales
}

\author{
Jeanne Talbot, MD, PhD; Jennifer L. Phillips, PhD; Pierre Blier, MD, PhD
}

\begin{abstract}
A growing body of literature has shown the effectiveness of ketamine for treating chronic depression. How long the beneficial effects of repeated ketamine last once infusions are stopped, however, remains largely unknown. Understanding the challenges that ensue after ketamine cessation can help clinicians optimally guide patients who opt for ketamine treatment and minimize the associated risks. In this commentary, we discuss some unexpected data gathered from participants of a pilot study on the effects of adjunctive ketamine infusion for resistant depression.
\end{abstract}

\section{Introduction}

There is a growing body of literature demonstrating the effectiveness of ketamine for the treatment of chronic depression. In patients with mood disorders, subanesthetic doses of intravenous ketamine have been shown to rapidly decrease depressive symptoms ${ }^{1}$ and suicidal ideation. ${ }^{2}$ Although the antidepressant effect of a single infusion of ketamine usually abates within a week, it can be prolonged with repeated administration. ${ }^{3}$ How long the beneficial effects of repeated ketamine last once infusions are stopped, however, remains largely unknown. With a few exceptions, ${ }^{4,5}$ most clinical trials have not followed patients beyond a short period of time after trial completion. This has perhaps obscured inadvertent, negative sequelae that may emerge in the weeks and months after ketamine cessation. Understanding these challenges can help clinicians optimally guide patients who opt for ketamine treatment and minimize risks.

Unexpected data about the clinical course of ketamine trial participants were gathered during a psychotherapy pilot study we conducted immediately after our 3-phase clinical trial that examined the effects of adjunctive ketamine infusions for refractory depression. ${ }^{6}$ Our preliminary study results were presented at the European Association of Cognitive Behavioural Therapy Annual Meeting in 2016. Two adverse events occurred that raise questions about the impact of receiving what is often perceived as a novel and end-ofthe-line treatment. Our post-trial pilot study examined whether the transient period of mood improvement achieved after a series of 6-10 ketamine infusions could be used to jump-start a behavioural activation treatment for depression. In fact, ongoing weekly psychotherapy sessions provided a unique opportunity to explore patients' percep- tions and interpretations of their depressive symptoms after participation in a ketamine trial.

A total of 13 participants were enrolled in the adjunct psychotherapy study. Both ketamine responders and nonresponders received 12-16 sessions of behavioural activation therapy after the trial of intravenous ketamine was completed. Throughout both studies, participants maintained their regimen of concomitant medications. During the course of the psychotherapy study, 1 participant made a suicide attempt, and 1 became acutely suicidal.

\section{Case 1}

Individual 1 was a 45-year-old professional with a 5-year history of depression. He remitted with ketamine and rapidly reengaged with his daughter and wife, and resumed hobbies and intellectual pursuits. During a 2-week period, he experienced moments of anhedonia and low mood. These symptoms were considerably worsened by the meaning he attributed to them: "I have had [electroconvulsive therapy] ECT, every medication and augmentation agent, and nothing has worked until ketamine, and that medication is not available to me now. I cannot go back to how I felt knowing there is no other option for me." Following a serious suicide attempt, his life was saved by quick-acting police officers.

\section{Case 2}

Individual 2 was a 32-year-old social worker. One month into the study, in the context of marital conflict, there was a drop in her mood that was further worsened because of her interpretation of her symptoms: "I realized that there were no other treatments for me and that I was going to be sick my

Correspondence to: P. Blier, Mood Disorders Research Unit, The Royal's Institute of Mental Health Research, 1145 Carling Ave., Ottawa, ON K1Z 7K4; Pierre.Blier@theroyal.ca

Submitted April 5, 2019; Revised June 12, 2019; Accepted June 14, 2019; Published online Oct. 1, 2019

DOI: $10.1503 / j p n .190073$ 
entire life like my father. I could not do this to my kids. I know they would be better off without me." Fortunately, she shared her acute suicidal thoughts, and additional support was provided during the crisis.

\section{Implications}

Our experience does not suggest that ketamine causes an increase in suicidal ideation or behaviour. In contrast, consistent with the literature, ${ }^{7}$ our data indicate a robust decrease in suicidal ideation while ketamine infusions continue. ${ }^{8}$ However, as with most antidepressant strategies, to maintain benefits reliably, ketamine needs to be administered beyond a short time period (i.e., 6 weeks, as in our study). It is possible that the 2 cases described above reflect a cautionary tale when treating individuals with a strongly promoted novel, short-acting treatment for depression that may then become unavailable to patients because of research study constraints or personal financial reasons. Indeed, the proposed price of intranasal esketamine recently approved by the U.S. Food and Drug Administration will preclude it as a treatment option for many. Presenting such fast-acting medications as an initial part of a more comprehensive treatment plan can mitigate the risk of patients catastrophically interpreting normal and expected waxing and waning mood symptoms as an imminent sign of relapse and the end of all hope.

Despite the excitement elicited by the possibility of ketamine therapy, we still lack guidelines for the long-term treatment of individuals following response to acute infusions. Thus far, nothing has been shown to prolong the effects of ketamine successfully, likely because of its novel mechanism of action. At this point, evidence from trials of esketamine suggests that the therapeutic benefits of ketamine can be maintained with continued administration at reduced frequencies. ${ }^{9}$ Clearly, the basic principles of good clinical management are not to be abandoned with a novel treatment option. Indeed, there are clear guidelines for the continued administration of conventional medications used to treat depression, even once a therapeutic response has been obtained. ${ }^{10}$ Continuing research is investigating the frequency of ketamine administration necessary to maintain its effects in the long term. Furthermore, as in other areas of medicine, the management of chronic illnesses is optimal when there is a comprehensive treatment plan. For example, in patients with diabetes, outcomes are improved with nutritional guidance, lifestyle change coaching and medication. Similarly, the treatment of chronic refractory depres- sion with ketamine is likely best optimized with adjunct psychosocial treatments. Ultimately, patients should be cautioned against pinning all their hopes on a single, novel pharmacological treatment and should be advised to engage in other empirically supported approaches.

Affiliations: From the The Royal's Institute of Mental Health Research, Mood Disorders Research Unit, Ottawa, Ont. (Talbot, Phillips, Blier); and the Department of Psychiatry, University of Ottawa, Ottawa, Ont. (Talbot, Phillips, Blier).

Competing interests: $P$. Blier declares receiving honoraria from Janssen for participating on its advisory board, giving lectures and preparing educational material. He is also the site principal investigator for esketamine trials (Janssen). J. Talbot and J. Phillips declare no competing interests.

Contributors: All authors contributed substantially to the conception, writing and revision of this article; approved the final version for publication; and can certify that no other individuals not listed as authors have made substantial contributions to the paper.

\section{References}

1. Sanacora G, Frye MA, McDonald W, et al. A consensus statement on the use of ketamine in the treatment of mood disorders. JAMA Psychiatry 2017;74:399-405.

2. Wilkinson ST, Ballard ED, Bloch $\mathrm{MH}$, et al. The effect of a single dose of intravenous ketamine on suicidal ideation: a systematic review and individual participant data meta-analysis. Am J Psychiatry 2018; 175:150-8.

3. Murrough JW, Perez AM, Pillemer S, et al. Rapid and longer term antidepressant effects of repeated ketamine infusions in treatmentresistant major depression. Biol Psychiatry 2013;74:250-6.

4. Shiroma PR, Johns B, Kuskowski M, et al. Augmentation of response and remission to serial intravenous subanaesthetic ketamine in treatment resistant depression. J Affect Disord 2014;155:123-9.

5. Zhan Y, Zhang B, Zhou Y, et al. A preliminary study of antisuicidal efficacy of repeated ketamine infusions in depression with suicidal ideation. J Affect Disord 2019;251:205-12.

6. Phillips JL, Norris S, Talbot J, et al. Single, repeated, and maintenance ketamine infusions for treatment-resistant depression: a randomized controlled trial. Am J Psychiatry 2019;176:401-9.

7. Price RB, Mathew SJ. Does ketamine have anti-suicidal properties? Current status and future directions. CNS Drugs 2015;29:181-8.

8. Phillips JL, Norris S, Talbot J, et al. Ketamine exerts a prolonged reduction in suicidal ideation independent of its antidepressant effects. Int J Neuropsychopharmacol 2016;19(Suppl 1):81.

9. Daly EJ, Singh JB, Fedgchin M, et al. Efficacy and safety of intranasal esketamine adjunctive to oral antidepressant therapy in treatment-resistant depression. A randomized clinical trial. JAMA Psychiatry 2018;75:139-48.

10. Kennedy SH, Lam RW, McIntyre RS, et al. Canadian Network for Mood and Anxiety Treatments (CANMAT) 2016 clinical guidelines for the management of adults with major depressive disorder: Section 3. Pharmacological treatments. Can J Psychiatry 2016;61:540-60. 